Am. J. Trop. Med. Hyg., 71(4), 2004, pp. 434-440

Copyright @ 2004 by The American Society of Tropical Medicine and Hygiene

\title{
THE EFFECTS OF SHORT-TERM IRON SUPPLEMENTATION ON IRON STATUS IN INFANTS IN MALARIA-ENDEMIC AREAS
}

\author{
CLARA MENENDEZ, DAVID SCHELLENBERG, LLORENÇ QUINTO, ELIZEUS KAHIGWA, LUISA ALVAREZ, \\ JOHN J. APONTE, AND PEDRO L. ALONSO \\ Center for International Health y Departamento de Bioquimica Clínica Institut d'Investigacions Biomèdiques August Pi i Sunyer \\ Hospital Clinic, Barcelona, Spain; Ifakara Health Research and Development Centre, Ifakara, Tanzania; St. Francis Designated \\ District Hospital, Ifakara, Tanzania
}

\begin{abstract}
Iron deficiency and Plasmodium falciparum malaria are the two main causes of anemia in young children in region endemic for this disease. The impact on iron status of prophylactic oral iron supplementation $(2 \mathrm{mg} / \mathrm{kg} / \mathrm{day}$ from two to six months of age) and the duration of this effect were assessed in a group of 832 Tanzanian infants exposed to $P$. falciparum malaria. Iron parameters and red blood cell indices were assessed at $2,5,8$, and 12 months of age. Infants who received iron supplements had a significantly lower prevalence of iron deficiency $(P<0.01$ at 5 months and $P<0.001$ at 8 and 12 months). Red blood cell indices (mean corpuscular volume, mean cell hemoglobin, and mean cell hemoglobin concentration) were increased in children receiving iron supplementation and they did not differ between those protected and unprotected against malaria. The prevalence of ferropenia was similar in children protected against malaria and in those who were not protected and did not receive iron supplements (34.7\% versus $37.3 \%$ at 12 months of age). We concluded that iron supplementation between the ages of 2-6 months improves iron status at least up to 12 months of age. Malaria infection does not contribute to iron deficiency.
\end{abstract}

\section{INTRODUCTION}

The burden of anemia on mortality and morbidity worldwide is difficult to quantify, but community prevalence figures reach $90 \%$ of the children being anemic in some sub-Saharan countries. Severe anemia in young children is one of the main reasons for admission to hospitals in many African countries, where it imposes a great demand on already limited resources. Malaria infection and iron deficiency, especially during the first year of life, are the two main causes of anemia in young children in malaria endemic areas. ${ }^{1-3}$

The high prevalence of iron deficiency ${ }^{4}$ in infants in developing countries is explained mainly by an inadequate iron intake, poor iron stores as a result of low birthweight, deficient maternal iron stores, and frequent infections. ${ }^{5,6}$ Despite guidelines recommending the delivery of iron to target groups, iron supplementation is not common practice in most developing countries, where iron is administered only for the management of anemia. The duration of iron supplementation required to prevent the development of ferropenic anemia in children in developing countries is not clear. Administration of iron supplements for more than 20 months has been recommended. ${ }^{7}$ However, there are major difficulties related to delivery of the supplements and maintenance of compliance for such a prolonged period. Assessing the duration of the effect on iron status of shorter and more feasible regimens of iron supplementation may be of public health relevance.

It has been suggested that malaria infection may also contribute to iron deficiency. The observation of an increased hematologic recovery when iron has been administered after a malaria episode ${ }^{8,9}$ may support this notion. Several mechanisms have been proposed for this, including a decreased iron absorption as a result of physiologic changes and malabsorption, ${ }^{10,11}$ reduced appetite, ${ }^{12}$ sequestration of iron within the malaria pigment hemozoin, ${ }^{13}$ and accumulation of iron within the cells of the reticuloendothelial system as a response to infection with Plasmodium falciparum. ${ }^{14}$ Conversely, adequate stores of iron have been found in the bone marrow of Gambian children with acute malaria attacks. ${ }^{13}$ It is therefore not yet clear whether malaria infection contributes to iron deficiency.

We have previously reported a randomized, placebocontrolled trial assessing the effects of malaria chemoprophylaxis and iron supplementation on anemia and malaria in infants. ${ }^{3}$ Malaria chemoprophylaxis reduced the incidence of anemia by approximately $60 \%$, while iron supplementation reduced this incidence by approximately $30 \%{ }^{3}$ Here we report the effects of these interventions on the prevalence of iron deficiency and on red blood cell indices throughout infancy. Within this framework, we have been able to test the hypothesis that malaria infection contributes to iron deficiency.

\section{MATERIALS AND METHODS}

Study area and population. The study was conducted in 1995-1996 in Ifakara in the Kilombero District of the Morogoro region of southeastern Tanzania. Characteristics of the study area have been described in detail elsewhere. ${ }^{3}$ The population of Ifakara is approximately 55,000 people, with the majority being subsistence farmers. Malaria transmission is intense and perennial with an entomologic inoculation rate in a nearby village of 300 infective bites per person per year. ${ }^{15}$ Malaria control relies on presumptive treatment of the clinical episode with an antimalarial (chloroquine at the time of the study). Government medical services in the town during the study period were limited to the 375-bed St. Francis Designated District Hospital and its adjacent maternal and child health clinic. ${ }^{16}$

Clinical malaria and severe anemia (packed cell volume [PCV] $<25 \%$ ) account for $37 \%$ and $35 \%$ of the admissions in children between one and five years of age and $44 \%$ and $41 \%$ in those less than one year of age $\mathrm{a}^{2}$ (Menendez $\mathrm{C}$, unpublished data). Malaria infection was found to be responsible for approximately $60 \%$ of severe anemia episodes, while iron deficiency accounted for approximately $30 \%$ of such episodes. ${ }^{3}$ The exact etiology of the $10 \%$ remaining severe anemia cases is unknown, although they are likely to be associated with bacterial and viral infections and micronutrient deficiencies 
other than iron deficiency. Hookworm infections are unlikely to play an important role as a cause of anemia in this group of children because $2(2.4 \%)$ of 205 children harbored hookworms in the feces in this setting. ${ }^{17}$

Study design. The study was part of a randomized, placebo-controlled, intervention trial of malaria chemoprophylaxis and iron supplementation for the prevention of malaria and anemia in infants. ${ }^{3}$ Eight hundred thirty-two children were randomized at two months of age to one of four intervention arms: 1) group IP = daily oral iron supplementation ( $2.4 \mathrm{~mL}$ of ferrous glycine sulfate $(2 \mathrm{mg} / \mathrm{kg}), 25 \mathrm{mg}$ of ferrous sulfate per $5 \mathrm{~mL}$, Plesmet syrup: Link Pharmaceuticals, Ltd., Bellshill, Lanarkshire, United Kingdom) plus weekly placebo; 2) group DP = weekly oral antimalarial chemoprophylaxis (2.5 mL of Deltaprim ${ }^{\circledR}, 3.125 \mathrm{mg}$ of pyrimethamine plus 25 $\mathrm{mg}$ of dapsone per $5 \mathrm{~mL}$; Wellcome Pty., Kempton Park, South Africa) plus daily placebo; 3 ) group $\mathrm{PP}=$ daily placebo and weekly placebo; and 4) group DI = daily iron and weekly chemoprophylaxis. Iron supplementation was administered from two to six months of age while malaria chemoprophylaxis was given from 2 to 12 months of age. Bottles containing iron supplementation were delivered at home by project field workers at enrollment and a second bottle was delivered two months later. Mothers administered the daily iron supplements at home. Compliance with supplementation was assessed by measuring the amount of syrup left in the bottle using a specially designed ruler at each weekly visit when the antimalarial was administered. Seventy-five percent of the children took more than $67 \%$ of the expected daily doses.

The frequency of both severe anemia and malaria episodes was assessed through a combination of passive case detection and cross-sectional surveys. Cross-sectional surveys were carried out at 2, 5, 8, and 12 months of age. At each crosssectional visit, finger prick blood samples were collected for hematologic and biochemical determinations and two thick and thin blood films were prepared for quantification of $P$. falciparum parasitemia and red blood cell morphology. The axillary temperature was measured and anthropometric parameters were recorded.

Infants found to have a PCV $<25 \%$ at any of the visits were withdrawn from the study and treated according to hospital guidelines consisting of a therapeutic course of an antimalarial drug plus daily oral iron in treatment doses for two weeks. Malaria episodes were treated following the Tanzanian national guidelines at the time of the study, which consisted of oral chloroquine ( $25 \mathrm{mg} / \mathrm{kg}$ over a three-day period) for uncomplicated cases or parenteral quinine for severe malaria cases. Blood transfusions were given following strict guidelines established at the hospital, which consisted of a PCV $<12 \%$ or the presence of signs of respiratory distress with a $\mathrm{PCV}<18 \%$.

The use of a placebo for iron supplements and malaria chemoprophylaxis was justified in this area of Tanzania for the following reasons. 1) Although there was some information on the prevalence of malaria infection in children in this area, no information existed with regards to its frequency in small infants or to its contribution to anemia and general morbidity. 2) Similarly, there was no information on the prevalence of iron deficiency in infants nor on its contribution to anemia in this age group in malaria endemic areas in general. 3) The efficacy of both low doses of iron supplementation and/or malaria chemoprophylaxis to prevent malaria and anemia in infants was unknown. 4) Finally, neither malaria chemoprophylaxis nor iron supplementation were (or are now) used for prevention of malaria and anemia in children and infants in malaria-endemic areas. This study excluded anemic children at the time of recruitment and treated those developing anemia or malaria according to the Tanzanian national treatment guidelines.

The study was explained to the parents or guardians of the participating children, their understanding of it was assessed with a set of standard questions, and written informed consent was obtained. The study received ethical clearance from the Tanzanian Commission for Science and Technology and from the Ethical Committee of the Hospital Clinic from Barcelona, Spain and adhered to the principles of the Declaration of Helsinki that governs biomedical research in human subjects.

Laboratory procedures. Blood samples were collected into microtainers containing EDTA and heparin (Becton Dickinson, Franklin Lakes, NJ) and the separated plasma was stored at $-20^{\circ} \mathrm{C}$. Variations in the volumes of plasma available precluded full analysis of all samples collected. Plasma ferritin was assayed by an immunoradiometric assay (Tandem-R Ferritin; Hybritech, Inc., San Diego, CA). The normal range for the kit for children of this age was $10-83 \mathrm{ng} / \mathrm{mL}$. Plasma iron (reference range $=11-150 \mu \mathrm{g} / \mathrm{dL}$ ) was measured colorimetrically using the ferrozine method without deproteinization in a Cobas Mira S analyzer (Roche Diagnostics, Basel, Switzerland). Plasma transferrin (reference range $=218-347 \mathrm{mg} / \mathrm{dL}$ ) was assayed by immunonephelometric methods using reagents in a nephelometer model A (Dade Behring, Marburg, Germany).

Red blood cell indices (mean corpuscular volume [MCV], mean cell hemoglobin [MCH], mean cell hemoglobin concentration $[\mathrm{MCHC}]$, and red blood cell distribution width [RDW]) were measured on blood collected into microtainers containing EDTA with a semiautomatic cell counter (Sysmex F800 microcell counter; TOA Medical Electronics, Kobe, Japan).

Light microscopes were used to count reticulocytes on thin blood films stained with methylene blue, prepared at the cross-sectional visit at 12 months of age in 100 randomly selected children.

Thick and thin blood films were stained with Giemsa for malaria parasites or with May-Grunwald for red blood cell morphology, respectively, and read with a light microscope following standard procedures. ${ }^{18}$ Briefly, each slide was read at least twice, and a third reading was done for major discrepancies between the results of the first two readings (either positive/negative discrepancies or a ratio of the readings $<0.66$ or $>1.33$ ). The majority reading for positive/negative discordances or the geometric mean of three positive readings was taken as the definitive result.

Definitions and statistical methods. Iron deficiency was defined as either 1) a plasma ferritin level $<12 \mathrm{ng} / \mathrm{mL}$ (children between two and five months old) or $<10 \mathrm{ng} / \mathrm{mL}$ (between 8 and 12 months old) or 2) a plasma iron level $<11 \mu \mathrm{g} / \mathrm{dL}$ and a transferrin concentration $>347 \mathrm{mg} / \mathrm{dL}$ for all ages. ${ }^{19}$

Microcytosis was defined as an MCV $<77 \mathrm{fl}$ (children between two and five months old) or $<70 \mathrm{fl}$ (children between 8 and 12 months old). Hypochromia was defined as an $\mathrm{MCH}$ value $<25 \mathrm{pg}$ or an $\mathrm{MCHC}$ value $<29 \mathrm{~g} / \mathrm{L}$. A low RDW was defined as value $<39 \mathrm{fl}$. Reticulocyte counts greater than $1 \%$ 
were considered increased according to the reference values published for infants. ${ }^{20}$ Severe anemia was defined as a PCV less than $25 \%$, and a malaria episode as an axillary temperature $\geq 37.5^{\circ} \mathrm{C}$ plus a $P$. falciparum asexual parasitemia of any density.

The red blood cell indices and iron parameters were compared with respect to the placebo-placebo group at each cross-sectional survey by using linear regression models for continuous variables. Logistic regression models were used to compare the prevalence of iron deficiency by intervention group and cross-sectional visit with respect to the placeboplacebo group. Analysis was done using Stata Statistical Software version 5.0 (Stata Corporation, College Station TX).

\section{RESULTS}

Baseline hematologic and iron parameters are shown in Table 1 . There were no statistically significant differences in any of the variables studied between the four intervention groups before the interventions started at two months of age. Eight hundred thirty-two children met the inclusion criteria to be randomized into one of the four groups. Throughout the study, 209 children were found to have severe anemia and were withdrawn from the study interventions. Other reasons for withdrawal were migration out of the area, parental refusal, and investigators request. There was no difference between the study groups in the number of children withdrawn for any of these reasons except for severe anemia. In this case, significantly more children in the placebo-placebo group had to be withdrawn and treated than in any of the other three groups. $^{3}$

Iron deficiency. The prevalence of iron deficiency by treatment group and cross-sectional survey is shown in Table 2. The statistical analysis was based only on those children who had each result and no attempt was made to predict missing values.

The prevalence of iron deficiency increased throughout the first year of life. There was a reduction of plasma iron and ferritin between two and five 5 months of age, which is consistent with the physiologic destruction of red blood cells during the first three months of life. The placebo group showed an increase in the prevalence of iron deficiency from less than $1 \%$ at two months of age to more than $30 \%$ by 12 months of age. The children who received only malaria chemoprophylaxis had similar prevalences of iron deficiency than those receiving no intervention. At all surveys after the intervention started, the frequency of iron deficiency was lower in the two groups receiving iron supplementation compared with the placebo-placebo group. Children who had received iron for four months during the first year of life had a lower (64-92\%) risk of iron deficiency than those who did not (Table 3). There were no significant differences in the frequency of iron deficiency between the two iron-supplemented groups.

Red blood cell indices and iron parameters by intervention group and age during the first year of life. Table 1 shows the values of biochemical markers of iron status and quantitative red blood cell indices by treatment group and survey during the intervention period. Plasma iron concentrations were higher during supplementation than after supplementation. Ferritin and transferrin concentrations remained significantly improved up to and including the 12 month survey. Red blood cell indices ( $\mathrm{MCV}, \mathrm{MCH}$, and $\mathrm{MCHC}$ ) were consistently higher in the two groups receiving iron supplements than in those infants who did not. The beneficial effects of iron supplementation between two and six months of age on $\mathrm{MCV}, \mathrm{MCH}$, and $\mathrm{MCHC}$ were also maintained until one year of age.

The prevalence of microcytosis was highest at five months of age $(69 \%)$, decreasing progressively up to 12 months of age $(45 \%)$. Children on iron supplements had lower prevalence of microcytosis at all ages than those in the placebo-placebo group. The majority of children had hypochromia from five months of age (89\%), with a peak prevalence at 12 months of age in all groups (96\%). Hypochromia was less frequent in children who received iron supplements compared with the placebo-placebo group, except at five months when only the group receiving iron alone had a lower prevalence of hypochromia compared with the placebo-placebo group, and this was of borderline significance $(P=0.06)$ (Table 2$)$.

The proportion of children with increased reticulocyte counts was higher in the two groups receiving iron supplements compared with the groups that did not receive iron, although the difference did not reach statistical significance (39.1\% and $38.5 \%$ in the IP and DI groups, respectively, compared with $13.0 \%$ and $32.0 \%$ in the PP and DP groups, respectively; $P=0.165)$. Neither iron nor malaria chemoprophylaxis with Deltaprim were associated with side effects as assessed through morbidity and analytical surveillance. ${ }^{3}$

\section{DISCUSSION}

In this area of sub-Saharan Africa, there is a high prevalence of iron deficiency even in infants less than six months of age. The prevalence of iron deficiency in our study increased with age in all intervention groups. More than one-third of children who did not receive iron supplements were iron deficient by one year of age. However, this may still be an underestimate judging by the prevalence of microcytosis and hypochromia. These high frequencies of both microcytosis and hypochromia may be partly explained by the high frequency of $\alpha$-thalassemia found in these children $(44.3 \%$ and $10.3 \%$ for heterozygous $[-\alpha / \alpha \alpha]$ and homozygous $[-\alpha /-\alpha /]$, respectively, Mayor A, unpublished data), which was equally distributed between the four intervention groups $(P=0.86)$. The recognized difficulties in assessing iron status in populations exposed to malaria may have contributed to this underestimation in the frequency of iron deficiency. ${ }^{12,21-23} \mathrm{~A}$ reliable, non-invasive tool for the assessment of iron deficiency in these populations remains elusive, since malaria infection affects all conventional laboratory tests of iron status. Measurement of soluble transferrin receptors has been proposed as an alternative test to overcome this problem. ${ }^{23}$ However, a recent study has showed that this is also modified by malaria parasitaemia. $^{24}$

Iron supplements were started at two months of age. At the time of the study, there were no clear recommendations regarding iron supplementation for the prevention of iron deficiency in infants in malaria-endemic areas. As in many other malaria-endemic regions, the prevalence of anemia is very high during the first year of life. This, together with the high prevalence of maternal anemia and of low birth weight, led us to start the administration of iron supplements earlier than 
IRON SUPPLEMENTATION FOR INFANTS IN MALARIA-ENDEMIC AREAS

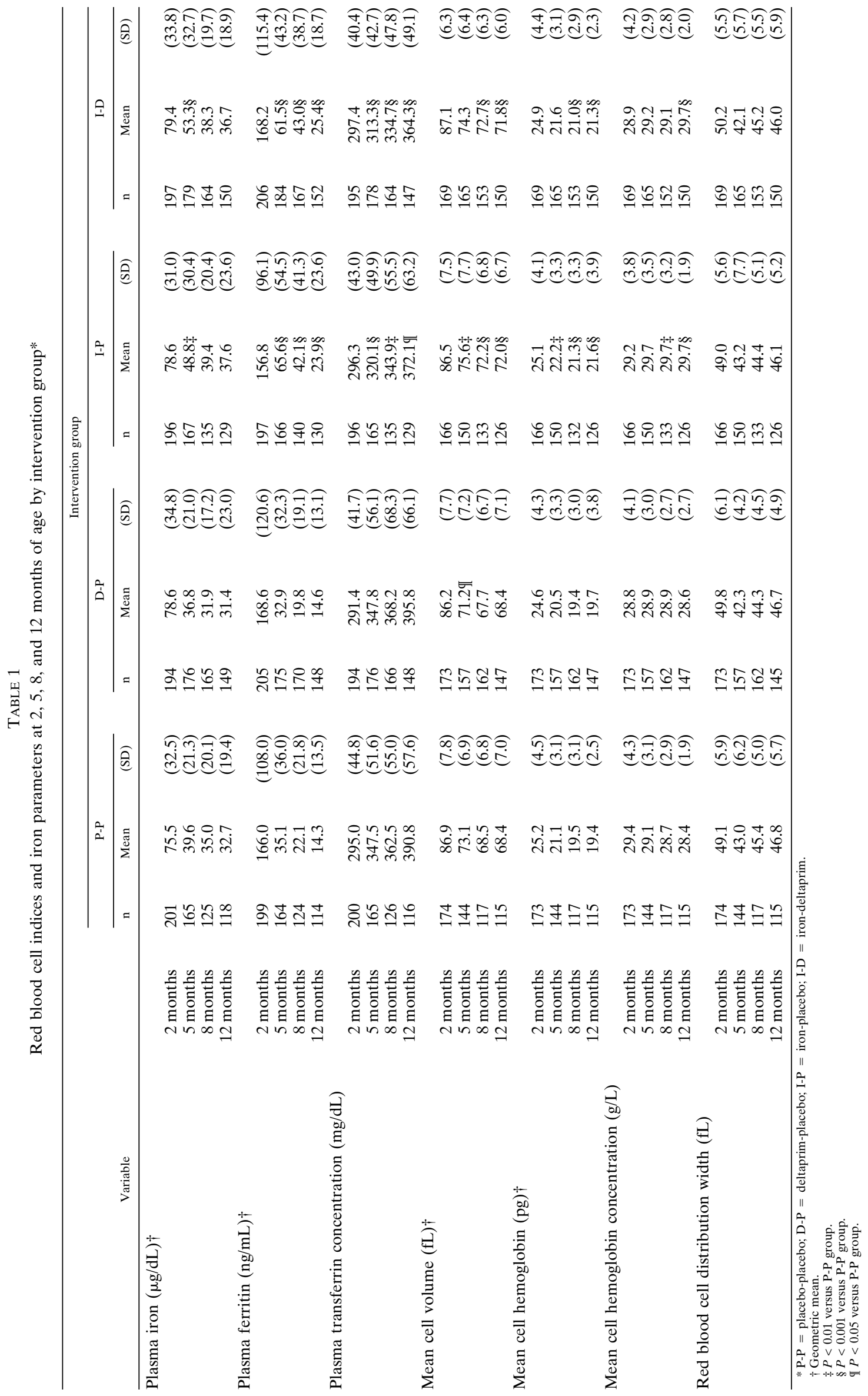




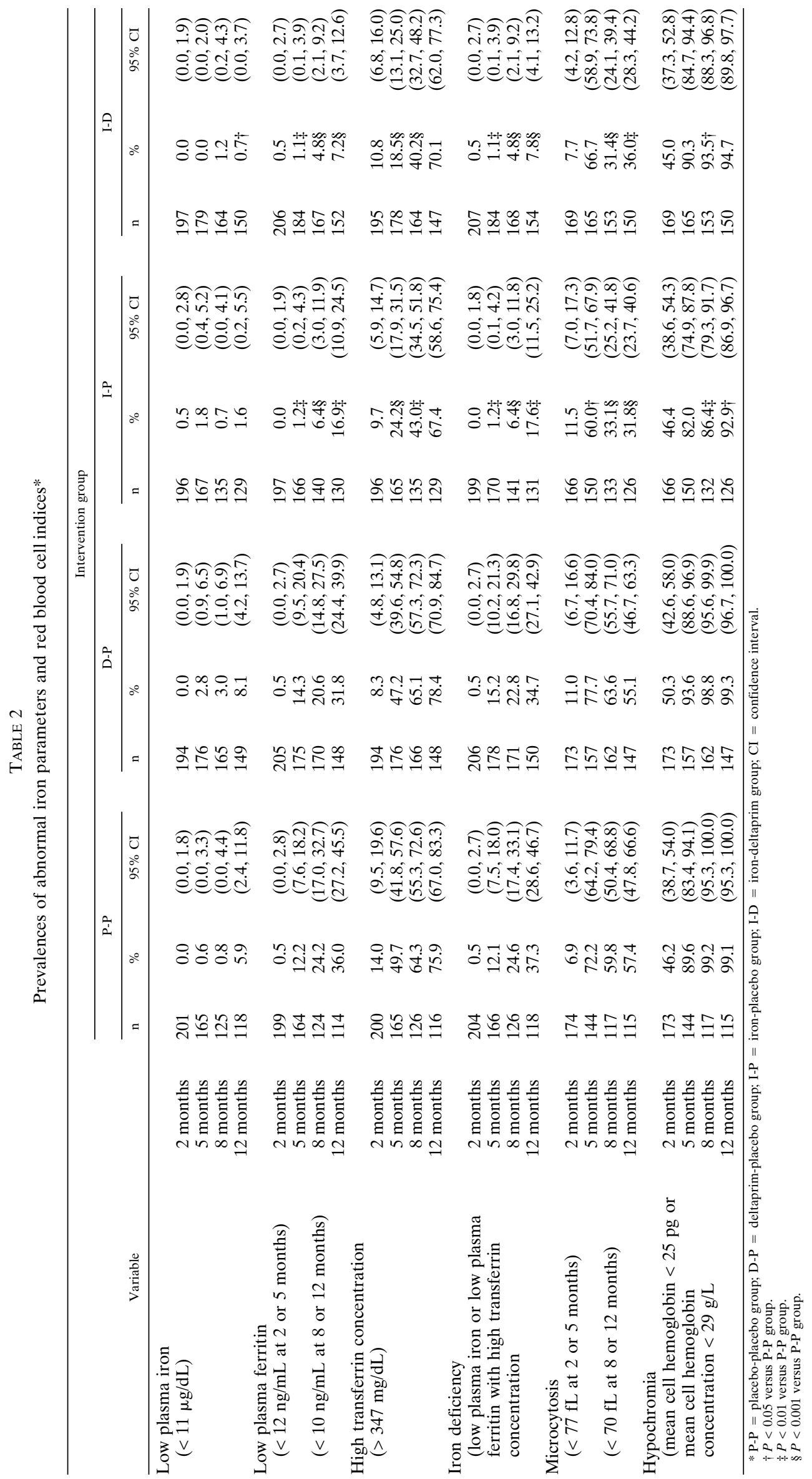


TABLE 3

Odds ratio of iron deficiency at each age by intervention group*

\begin{tabular}{|c|c|c|c|c|c|c|c|c|}
\hline \multirow[b]{3}{*}{ Intervention group } & \multicolumn{8}{|c|}{ Age (months) } \\
\hline & \multicolumn{2}{|c|}{2} & \multicolumn{2}{|c|}{5} & \multicolumn{2}{|c|}{8} & \multicolumn{2}{|c|}{12} \\
\hline & $\mathrm{OR}$ & $(95 \% \mathrm{CI})$ & OR & $(95 \% \mathrm{CI})$ & OR & $(95 \% \mathrm{CI})$ & OR & $(95 \% \mathrm{CI})$ \\
\hline P-P & 1 & & 1 & & 1 & & 1 & \\
\hline D-P & 1.0 & $(0.1,15.9)$ & 1.3 & $(0.7,2.4)$ & 0.9 & $(0.5,1.6)$ & 0.9 & $(0.5,1.5)$ \\
\hline $\mathrm{I}-\mathrm{P}$ & $-\dagger$ & & $0.1 \ddagger$ & $(0.0,0.4)$ & $0.2 \S$ & $(0.1,0.5)$ & 0.4 必 & $(0.2,0.6)$ \\
\hline I-D & 1.0 & $(0.1,15.9)$ & $0.1 \ddagger$ & $(0.0,0.4)$ & $0.2 \S$ & $(0.1,0.4)$ & $0.1 \S$ & $(0.1,0.3)$ \\
\hline
\end{tabular}

usually recommended for non-malaria-endemic areas. The duration of supplementation was chosen to maximize compliance. Iron supplementation for a relatively short period of time, between two and six months of age, resulted in significant improvements in iron status sustained at least up to one year of age. A higher proportion of children with increased reticulocyte counts at 12 months of age in the groups who had received iron supplements compared with the ones who did not suggests a more active erythroid marrow in those children. Taken together with the efficacy results previously reported, ${ }^{3}$ these results suggest that short-term iron supplementation regimens improve iron status in a clinically and hematologically significant way and for a prolonged period. Furthermore, it is possible that this regimen of iron supplementation might be delivered at the time of existing health contacts, such as with the Expanded Program of Immunization.

Long-term (18-22 months) iron supplementation regimens have been proposed for the prevention of iron deficiency in children living in areas where anemia is highly prevalent. ${ }^{7}$ This recommendation is based on empirical hematologic benefits. However, it remains unclear whether such long-term supplementation might increase the risk of malaria infection. In addition, the effectiveness of this intervention clearly relies on good compliance, as well as on an available and sustainable supply of iron syrup and an effective means of delivering the supplements to the target group.

Finally, the results of this randomized, placebo-controlled trial do not suggest that malaria contributed to the high prevalence of iron deficiency. Children who received malaria chemoprophylaxis were partially protected against malaria infection, as was shown by a statistically significantly lower incidence of malaria episodes in this group compared with those not receiving antimalarial prophylaxis. ${ }^{3}$ However, they had similar prevalences of iron deficiency at all surveys as the children who were not protected against malaria and who did not receive iron supplements. This is unlikely to be a chance finding because a similar pattern was observed for microcytosis and hypochromia, neither of which were included in the definition of iron deficiency. The possibility that dapsonerelated hemolysis worsened hematologic parameters in children receiving chemoprophylaxis is excluded by the lack of a difference in red blood cell indices between the two ironsupplemented groups.

In summary, this study shows that a potentially practical iron supplementation regimen between two and six months of age produced significant and sustained improvements in iron status at least up to the end of the first year of life. Although iron deficiency was not eliminated in study children, its prevalence was dramatically reduced. Studies are warranted to assess the longer-term effects of this approach to iron supplementation and to assess the feasibility of delivering supplements to the target group at routine health contacts.

Received May 29, 2003. Accepted for publication February 18, 2004.

Acknowledgments: We are indebted to the parents and guardians of the children involved in the study. We thank the staff of the Ifakara Health Research and Development Centre and the St. Francis Designated District Hospital, Dr. F. Lwilla, and the District Health Management Team for their support in facilitating the study team to carry out the study. We are also grateful to Dr. R. Fernandez and the staff of the Nuclear Medicine Laboratory of the Hospital Clinic for testing the plasma ferritins and to Professor Alan Fleming for his comments on the manuscript.

Financial support: This study was supported by grants from the UNDP/World Bank/World Health Organization Special Program for Research and Training in Tropical Diseases, the Spanish Agency for International Cooperation, and the Fondo de Investigaciones Sanitarias. The Ifakara Health Research and Development Centre and St. Francis Designated District Hospital receive funding from the Swiss Agency for Development and Cooperation and the Government of Tanzania.

Author's addresses: Clara Menendez, David Schellenberg, Llorenç Quinto, Luisa Alvarez, John J. Aponte, and Pedro L. Alonso, Center for International Health, Hospital Clinic/University of Barcelona, Villarroel 170, E-08036, Barcelona, Spain, Telephone: 34-93-2275706, Fax: 34-93-451-5272, E-mails: cmenende@medicina.ub.es, DMSchellenberg@aol.com, llquinto@medicina.ub.es, lalvarez@clinic. ub.es, Japonte@medicina.ub.es, and alonso@medicina.ub.es. Elizeus Kahigwa, Ifakara Research and Development Center, Ifakara Tanzania and St. Francis District Designated Hospital, Ifakara, Tanzania. E-mail: ekahigwa@yahoo.com.

\section{REFERENCES}

1. Fleming AF, 1981. Iron deficiency in the tropics. Clin Haematol 11: $365-388$.

2. Schellenberg D, Menendez C, Kahigwa E, Font F, Galindo C, Acosta C, Armstrong-Schellenberg J, Aponte JJ, Kimario J, Urassa H, Mshinda H, Tanner M, Alonso P, 1999. African children with malaria in an area of intense Plasmodium falciparum transmission: features on admission to the hospital and risk factors for death. Am J Trop Med Hyg 61: 431-438.

3. Menendez, C, Kahigwa E, Hirt R, Vounatsou P, Aponte JJ, Font F, Acosta CJ, Schellenberg DS, Galindo C M, Kimario J, Urassa H, Brabin B, Smith TA, Kitua AY, Tanner M, Alonso PL, 1997. Randomised placebo-controlled trial of iron supplementation and malaria chemoprophylaxis for prevention of severe anemia and malaria in Tanzanian infants. Lancet 350: 844-850.

4. Provan D, 1999. Mechanisms and management of iron deficiency anemia. Br J Haematol 105: 19-26. 
5. Oski FA, 1993. Current concepts: iron deficiency in infancy and childhood. N Eng J Med 329: 190-193.

6. Brabin B, 1992. Fetal anemia in malarious areas: its causes and significance. Ann Trop Paediat 12: 303-310.

7. Stoltzfus R, Dreyfuss ML, 1998. Guidelines for the Use of Iron Supplements to Prevent and Treat Iron Deficiency Anemia. Washington, DC: INACG/WHO/UNICEF, ILSI Press.

8. Bojang KA, Palmer A, Van Hensbroek MB, Banya WAS, Greenwood BM, 1997. Management of severe malarial anemia in Gambian children. Trans R Soc Trop Med Hyg 91: 557-561.

9. Van Hensbroek MB, Morris-Jones S, Meisner S, Jaffar S, Bayo L, Dackour R, Phillips C, Greenwood BM, 1995. Iron, but not folic acid, combined with effective antimalarial therapy promotes haematological recovery in African children after acute falciparum malaria. Trans R Soc Trop Med Hyg 89: 672-676.

10. Willcox MC, Bjorkman A, Brohult J, 1985. The effect of persistent malarial infection on haemoglobin A2 levels in Liberian children. Trans $R$ Soc Trop Med Hyg 79: 242-245.

11. Molyneux MES, Looareesuwan IS, Menzies SL, Grainger SL, Phillips RE, Wattanagoon Y, Thompson RP, Warrell DA, 1989. Reduced hepatic blood flow and intestinal malabsorption in severe falciparum malaria. Am J Trop Med Hyg 40: 470-476.

12. Filteau SM, Tomkins AM, 1994. Micronutrients and tropical infections. Trans R Soc Trop Med Hyg 88: 1-3.

13. Abdalla SH, 1990. Iron and folate status in Gambian children with malaria. Ann Trop Paediat 10: 265-272.

14. Weinberg ED, 1978. Iron and infection. Microbiol Rev 42: 45-66.

15. Smith T, Charlwood JD, Kihonda J, Mwankusye S, Billingsley P, Muwissen J, Lyimo E, Takken W, Teuscher T, Tanner M, 1993. Absence of seasonal variation in malaria parasitaemia in an area of intense seasonal transmission. Acta Trop 54: 55-72.

16. Schellenberg D, Aponte J, Kahigwa E, Mshinda H, Tanner M, Menendez C, Alonso PL, 2003. The incidence of clinical ma- laria detected by active case detection in children in Ifakara, southern Tanzania. Trans R Soc Trop Med Hyg 97: 1-8.

17. Gascon J, Vargas M, Schellenberg D, Urassa H, Casals C, Kahigwa E, Aponte JJ, Mshinda H, Vila J, 2000. Diarrhea in children under 5 years of age from Ifakara, Tanzania: a casecontrol study. J Clin Microbiol 38: 4459-4462.

18. Alonso PL, Smith T, Armstrong Schellenberg JRM, Masanja H, Mwankusye S, Urassa H, Bastos de Azevedo I, Chongela J, Kobero S, Menendez C, Hurt N, Thomas MC, Lyimo E, Weiss N, Hayes R, Kitua A, Lopez MC, Kilama WC, Teuscher T, Tanner M, 1994. Randomised trial of efficacy of SPf66 vaccine against Plasmodium falciparum malaria in children in southern Tanzania. Lancet 344: 1175-1181.

19. Meites S, 1989. Reference (normal) values. Meites S, ed. Pediatric Clinical Chemistry. Third edition. Washington, DC: Amercian Association for Clinical Chemistry Press, 119-120.

20. Dacie JV, Lewis SM, 1995. Reference ranges and normal values. Dacie JV, Lewis SM, eds. Practical Haematology. Eighth edition. Edinburgh: Churchill Livingstone, 17.

21. Das BS, Thurnham DI, Das DB, 1997. Influence of malaria on markers of iron status in children: implications for interpreting iron status in malaria-endemic communities. Br J Nutr 78: 751760.

22. Ayatse JO, Ekanem EE, 1994. Plasmodium falciparum malaria: its effects on some haematological parameters in normal and sickle cell Nigerian children. Trop Med Parasitol 45: 219-222.

23. Ferguson BJ, Skikne BS, Simpson KM, Baynes RD, Cook JD, 1991. Serum transferrin receptor distinguishes the anemia of chronic disease from iron deficiency anemia. J Lab Clin Med 119: 385-390.

24. Menendez C, Quinto LL, Kahigwa E, Alvarez L, Fernandez R, Gimenez N, Schellenberg D, Tanner M, Alonso PL, 2001. Malaria infection increases soluble transferrin receptors in Tanzanian infants. Am J Trop Med Hyg 65: 138-142. 